\title{
Numerical Simulation of Hydraulic Fracturing Based on the Extended Finite Element Method
}

\author{
Xiaogang $\mathrm{Li}^{1,2}$, Liangping $\mathrm{Yi}^{2, *}$, Changyin $\mathrm{Liu}^{1}$, Zhiyu Sun ${ }^{1}$ and Zhaozhong Yang ${ }^{2}$ \\ ${ }^{1}$ State Key Laboratory of shale oil gas accumulation mechanism and effective development, SINOPEC Beijing 100083, China \\ ${ }^{2}$ Petroleum and Natural Gas Engineering Institute, Southwest Petroleum University, Xindu Avenue 8, $610500 \mathrm{Chengdu,}$ \\ China \\ ${ }^{*}$ Corresponding author
}

\begin{abstract}
This paper proposed a numerical model to simulate the hydraulic fracture propagation based on the extended finite element method(XFEM), in which a fracture representation is explicit and independent of the mesh grid. The fully coupled formulation described various physical phenomena, including the solid skeleton deformation as well as the fluid flow in porous medium, along the fracture and through the fracture sides toward the surrounding porous medium. The interactive integral method is applied to calculate the stress intensity factor in consideration of fluid pressure on fracture faces. The maximum circumferential stress criterion is used for identifying the fracture growth condition and orientation. In comparison with the current existing XFEM models for hydraulic fracturing, this approach neither needs to introduce leak-off coefficient to describe the fluid leak-off phenomenon, nor requires to assume fracture propagation orientation. Data indicates that the model is valid through a comparison of the model numerical solutions with the semi-analytical(KGD) solution . In the end, fractures simultaneously propagate from three perforating clusters in the horizontal well is simulated at the end of this paper.
\end{abstract}

Keywords-extended finite element method; hydraulic fracture propagation; the interactive integral; horizontal well

\section{INTRODUCTION}

Hydraulic fracturing has captured considerable attention in that it is widely used in engineering areas, including enhancing hydrocarbon production and geothermal energy extraction $[1,2]$ and measurement of in-situ stresses[3,4], there are mainly four physical processes in hydraulic fracturing : the flow of the fracturing fluid within the fracture, the leak-off of the fracturing fluid from the fracture into the surrounding porous medium and flooding the pore fluid through the surrounding porous medium, mechanical deformation induced by fluid pressurizing, and fracture propagation. Those four processes are coupled with each other. The extended finite element method (XFEM), first brought forward by Belytschko and Black[5], is one of the most effective methods to solve fracture propagation problem[6]. There have been numerous studies on fluid-driven fracture propagation based on XFEM.

Brice Lecampion[7] presented a XFEM for the solution of hydraulic fracture problems. Lamb et al.[8] presented a fracture mapping approach combined with the XFEM to simulate coupled deformation and fluid flow in fractured porous media, however, they both neglecting the fracture propagation.
Ren et al.[9] presented an XFEM algorithm for hydraulic fracturing analysis with considering water pressure on the crack surface. Gordeliy and Peirce[10] described coupled algorithms that use the XFEM to solve the elastic crack component of the elasto-hydrodynamic equations that govern the propagation of hydraulic fractures in an elastic medium. But both of them ignored the filtration of fracturing fluid.

Dahi-Taleghani [11] developed an XFEM algorithm to simulate fracture propagation, initiation and intersection in fractured reservoirs. Zhou et al.[12]combined the extended finite element and the finite volume methods to model hydraulic-driven fractures with arbitrary orientation in tight gas reservoirs. Wang[13] presented a fully coupled hydraulic fracture propagation model based on the extended finite element method, cohesive zone method and Mohr-Coulomb theory of plasticity. However, their algorithm need to introduce leak-off coefficient to describe the fluid leak-off phenomenon.

Carrier et al.[14] developed a zero-thickness finite element to model the hydraulic fracture, the fracture propagation is governed by a cohesive zone model and the flow within the fracture by the lubrication equation. Mohammadnejad and Khoei [15] carried out the extended finite element modeling of cohesive crack propagation in multiphase porous media, and they[16]subsequently proposed a fully coupled numerical model for the modeling of the hydraulic fracture propagation in porous media using the extended finite element method in conjunction with the cohesive crack model. Meschke and Leonhart[17] presented a numerical model to simulate the hydraulic fracture propagation based on the extended finite element method, in this model the enrichment functions are space and time variant. Although the above model not need to introduce leak-off coefficient to describe the fluid leak-off phenomenon, they are all assume that the fracture along the straight line extension.

Dahi-Taleghani and Olson[18] use XFEM to study the interaction between pre-existing natural fracture and the advancing hydraulic fracture, and they [19] subsequently extend the numerical analysis of hydraulic-fracture/naturalfracture interaction to the case of cemented natural fractures. Khoei[20] also use the XFEM to simulate the mechanism of interaction between the hydraulic fracturing and frictional natural fault. But they all consider the rock as impermeable media. 
This paper presents a numerical model to simulate the hydraulic fracture propagation based on the extended finite element method. In comparison with the existing XFEM models for hydraulic fracturing, this approach neither needs to introduce leak-off coefficient to describe the fluid leak-off phenomenon, nor requires to assume fracture propagation orientation.

\section{GOVERNING EQUATIONS}

\section{A. Descriptions of Physical Model}

The two-dimensional domain $\Omega$ bounded by the outer boundary $\Gamma$ and the interior boundary $\Gamma_{\mathrm{f}}$, the discontinuity $\Gamma_{\mathrm{f}}$ reflects the hydraulic fracture. Because the width of the fracture is far shorter than its length, the fracture can be regarded as a discontinuity line in the view of the whole domain[21]. But on a local scale the fracture is twodimensional domain $\Omega f$ and bounded by $\Gamma_{\mathrm{f}}^{+} \cup \Gamma_{\mathrm{f}}^{-} \cup \Gamma_{\mathrm{w}}$, the boundary $\Gamma_{\mathrm{w}}$ represents the wellbore, as clarified in Fig.1.The fluid flow inside both matrix domain and hydraulic fracture domain is regarded as single-phase, and Darcy's law obeyed.

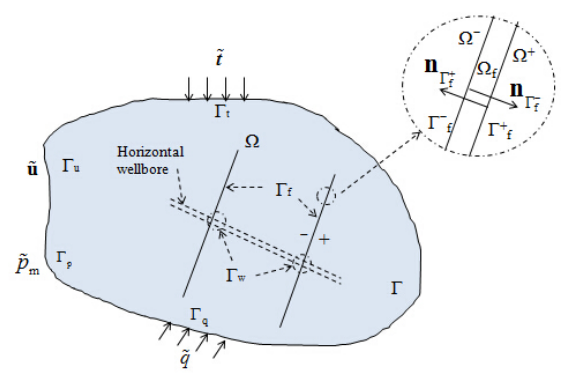

FIGURE I. DESCRIPTIONS OF PHYSICAL MODEL AND BOUNDARY CONDITIONS

\section{B. Strong Form}

The strong form of governing equations for the force equilibrium of the porous medium, fluid flow inside both matrix domain and fracture domain is demonstrated in this section. Hydraulic fracturing can be considered as a quasistatic problem[12], therefore the domain's force equilibrium equation can be written as

$$
\nabla \cdot \sigma=0
$$

The relationship between stress and strain can be expressed by mechanical constitutive Eq.(2), while the continuum condition inside the domain can be described by the linear displacement-strain continuum Eq.(3)

$$
\begin{aligned}
& \boldsymbol{\sigma}=\mathrm{D} \boldsymbol{\varepsilon} \\
& \boldsymbol{\varepsilon}=\mathrm{L} \mathbf{u}
\end{aligned}
$$

where $\sigma$ is stress $(\mathrm{Pa}), \varepsilon$ is the strain, D is elastic matrix , for plane strain state D can be described by Eq.(4), and L is differential operator can be described by Eq.(5)

$$
\mathrm{D}=\frac{E(1-v)}{(1-2 v)(1+v)}\left[\begin{array}{ccc}
1 & \frac{v}{1-v} & 0 \\
\frac{v}{1-v} & 1 & 0 \\
0 & 0 & \frac{1-2 v}{2(1-v)}
\end{array}\right]
$$

$$
\mathrm{L}=\left[\begin{array}{cc}
\frac{\partial}{\partial x} & 0 \\
0 & \frac{\partial}{\partial y} \\
\frac{\partial}{\partial y} & \frac{\partial}{\partial x}
\end{array}\right]
$$

where $E$ is young's modulus $(\mathrm{Pa}), v$ is Poisson's ratio.

According to the law of mass conservation, the continuity equation for the fluid flow in the permeable porous medium is written as

$$
\left(\frac{\alpha_{\mathrm{m}}-\phi}{K_{\mathrm{s}}}+\frac{\phi}{K_{\mathrm{w}}}\right) \frac{\partial p_{\mathrm{m}}}{\partial t}+\nabla \cdot\left(-\frac{\mathbf{K}_{\mathrm{m}}}{\mu} \nabla p_{\mathrm{m}}\right)=0
$$

Similarly, the continuity equation for flow inside the fracture domain is given by

$$
\frac{1}{K_{\mathrm{w}}} \frac{\partial p_{\mathrm{f}}}{\partial t}+\nabla \cdot\left(-\frac{\mathbf{K}_{\mathrm{f}}}{\mu} \nabla p_{\mathrm{f}}\right)+\nabla \cdot \dot{\mathbf{u}}=0
$$

where $\alpha_{\mathrm{m}}$ is the Biot coefficient, $\phi$ is the porosity of matrix rock, $K_{\mathrm{s}}$ is the bulk modulus of solid phase $(\mathrm{Pa}), K_{\mathrm{w}}$ is the bulk modulus of pore fluid( $(\mathrm{Pa}), \mathbf{K}_{\mathrm{m}}$ is the permeability tensor of matrix $\left(\mathrm{m}^{2}\right), p_{\mathrm{m}}$ is the pore fluid pressure in matrix $(\mathrm{Pa}), p_{\mathrm{f}}$ is the fluid pressure in fracture (Pa), $\mu$ is the fluid viscosity $(\mathrm{Pa} . \mathrm{s}), \mathbf{K}_{\mathrm{f}}$ is the permeability of fracture $\left(\mathrm{m}^{2}\right)$, and $\mathbf{K}_{\mathrm{f}}$ is given by[32]

$$
\mathbf{K}_{\mathrm{f}}=\frac{w^{2}}{12 \mathrm{f}}
$$

where $w$ is the width of fracture $(\mathrm{m})$, and $\mathrm{f}$ is the parameter accounting for the effect of the deviation from the ideal parallel fracture, ranging from 1.04 to 1.65 .

\section{Boundary Conditions}

In order to derived the weak form of governing equations, the boundary conditions must be given .The Dirichlet and Neumann boundary conditions are imposed on the external and inner boundary of the matrix are given by Eq.(9) and Eq.(10), respectively. 


$$
\begin{aligned}
& \left\{\begin{array}{l}
\mathbf{u}=\tilde{\mathbf{u}} \text { on } \Gamma_{\mathrm{u}} \\
p_{\mathrm{m}}=\tilde{p}_{\mathrm{m}} \text { on } \Gamma_{\mathrm{p}} \\
\boldsymbol{\sigma} \cdot \mathbf{n}_{\Gamma}=\tilde{\boldsymbol{t}} \text { on } \Gamma_{\mathrm{t}} \\
\left(-\frac{\mathbf{K}_{\mathrm{m}}}{\mu} \nabla p_{\mathrm{m}}\right) \cdot \mathbf{n}_{\Gamma}=\tilde{q} \text { on } \Gamma_{\mathrm{q}}
\end{array}\right. \\
& \left\{\begin{array}{c}
p_{\mathrm{m}}=\tilde{p}_{\mathrm{f}} \text { on } \Gamma_{\mathrm{f}} \\
\left(-\frac{\mathbf{K}_{\mathrm{m}}}{\mu} \nabla p_{\mathrm{m}}\right) \cdot \mathbf{n}_{\Gamma_{\mathrm{f}}}=-\tilde{q}_{\mathrm{f}} \text { on } \Gamma_{\mathrm{f}} \\
\boldsymbol{\sigma} \cdot \mathbf{n}_{\Gamma_{\mathrm{f}}}=-p_{\mathrm{f}} \cdot \mathbf{n}_{\Gamma_{\mathrm{f}}} \text { on } \Gamma_{\mathrm{f}}
\end{array}\right.
\end{aligned}
$$

where $\tilde{\mathbf{u}}$ is the prescribed displacement applied on the boundary $\Gamma_{\mathrm{u}}(\mathrm{m}), \tilde{p}_{\mathrm{m}}$ is the prescribed pressure imposed on the boundary $\Gamma_{\mathrm{p}}(\mathrm{Pa}), \tilde{\boldsymbol{t}}$ is the prescribed traction imposed on the boundary $\Gamma_{\mathrm{t}}(\mathrm{Pa}), \tilde{q}$ is the prescribed inflow rate of the fluid applied on the boundary $\Gamma_{\mathrm{q}}\left(\mathrm{m}^{3} / \mathrm{s}\right), \mathbf{n}_{\Gamma}$ is the unit outward normal vector to the external boundary $\Gamma, \tilde{p}_{\mathrm{f}}$ is the prescribed pressure imposed on the boundary $\Gamma_{\mathrm{f}}(\mathrm{Pa}), \tilde{q}_{\mathrm{f}}$ is the fluid exchange rate between the fracture and the surrounding porous medium on the boundary $\Gamma_{\mathrm{f}}\left(\mathrm{m}^{3} / \mathrm{s}\right), \mathbf{n}_{\Gamma_{\mathrm{f}}}$ is the unit outward normal vector to the inner boundary $\Gamma_{\mathrm{f}}$.

The boundary conditions are imposed on the fracture are given by Eqs.(11)

$$
\left\{\begin{array}{l}
\left(-\frac{\mathbf{K}_{\mathrm{f}}}{\mu} \nabla p_{\mathrm{f}}\right) \cdot\left(-\mathbf{n}_{\Gamma_{\mathrm{f}}}\right)=\tilde{q}_{\mathrm{f}} \quad \text { on } \quad \Gamma_{\mathrm{f}} \\
\left(-\frac{\mathbf{K}_{\mathrm{f}}}{\mu} \nabla p_{\mathrm{f}}\right) \cdot \mathbf{n}_{\Gamma_{w}}=-\tilde{q}_{\mathrm{w}} \quad \text { on } \Gamma_{w}
\end{array}\right.
$$

where $\mathbf{n}_{\Gamma_{w}}$ is the unit outward normal vector to the boundary $\Gamma_{\mathrm{w}}, \tilde{q}_{\mathrm{w}}$ is the prescribed inflow rate of the fluid applied on the boundary $\Gamma_{\mathrm{w}}\left(\mathrm{m}^{3} / \mathrm{s}\right)$.

\section{Weak Form}

According to the above strong-form equations and boundary conditions, weak-form equations can be derived based on the weighted residual method.

The weak form of the equilibrium equation for the matrix is obtained as

$$
\int_{\Omega} \delta \boldsymbol{\varepsilon}^{\mathrm{T}} \boldsymbol{\sigma} d \Omega-\int_{\Gamma_{\mathrm{f}}} \llbracket \delta \mathbf{u} \rrbracket^{\mathrm{T}} \mathbf{R}^{\mathrm{T}} \mathbf{I}_{\mathrm{f}}^{\mathrm{n}} p_{\mathrm{f}} d \Gamma=\int_{\Gamma_{\mathrm{u}}^{\mathrm{t}}} \delta \mathbf{u}^{\mathrm{T}} \tilde{\mathbf{t}} d \Gamma
$$

where $\mathbf{n}_{\Gamma_{\mathrm{f}}^{+}}$and $\mathbf{n}_{\Gamma_{\mathrm{f}}^{-}}$are the unit normal vectors directed to $\Omega_{\mathrm{f}}^{-}$ and $\Omega^{+}$, respectively, as clarified in Fig. 1 , and $\Gamma_{f}^{+}$and $\Gamma_{\mathrm{f}}^{-}$ represent the two sides of the discontinuity, the notation $\llbracket * \rrbracket=*^{+}-*^{-}$represents the difference between the corresponding values at the two fracture faces, $\delta \boldsymbol{\varepsilon}$ and $\delta \mathbf{u}$ both are the variation function, $\mathbf{R}$ is the coordinate transform matrix expressed as Eq.(13), $\mathbf{I}_{\mathrm{f}}$ is the unit normal vector of the fracture that expressed as Eq.(14).

$$
\begin{gathered}
\mathbf{R}=\left[\begin{array}{cc}
\cos \beta & \sin \beta \\
-\sin \beta & \cos \beta
\end{array}\right] \\
\mathbf{I}_{\mathrm{f}}^{\mathrm{n}}=\left[\begin{array}{ll}
0 & 1
\end{array}\right]^{\mathrm{T}}
\end{gathered}
$$

The weak forms of equations for flow in matrix and fracture can be expressed as Eq.(15).

$$
\begin{aligned}
& \int_{\Omega} \mathbf{w}_{\mathrm{p}}^{\mathrm{T}}\left(\frac{\alpha_{\mathrm{m}}-\phi}{K_{\mathrm{s}}}+\frac{\phi}{K_{\mathrm{w}}}\right) \frac{\partial p_{\mathrm{m}}}{\partial t} d \Omega+\int_{\Omega}\left(\nabla \mathbf{w}_{\mathrm{p}}\right)^{\mathrm{T}}\left(\frac{\mathbf{K}_{\mathrm{m}}}{\mu} \nabla p_{\mathrm{m}}\right) d \Omega+ \\
& \left.\int_{\Gamma_{\mathrm{f}}} \mathbf{w}_{\mathrm{p}}^{\mathrm{T}} \frac{w}{K_{\mathrm{w}}} \frac{\partial p_{\mathrm{f}}}{\partial t} d \Gamma+\int_{\Gamma_{\mathrm{f}}} w \frac{\mathbf{K}_{\mathrm{f}}}{\mu} \frac{\partial \mathbf{w}_{\mathrm{p}}^{\mathrm{T}}}{\partial x^{\prime}} \frac{\partial p_{\mathrm{f}}}{\partial x^{\prime}} d \Gamma+\int_{\Gamma_{\mathrm{f}}} \mathbf{w}_{\mathrm{p}}^{\mathrm{T}} \llbracket \dot{\mathrm{u}}_{y^{\prime}}\right] d \Gamma-\int_{\Gamma_{\mathrm{w}}} \mathbf{w}_{\mathrm{p}}^{\mathrm{T}} \tilde{q}_{\mathrm{w}} d \Gamma=0
\end{aligned}
$$

where $\mathbf{w}_{\mathrm{p}}$ is the weight function for fluid pressure.

\section{E. Discrete the Governing Equations by XFEM}

In the classic FEM, the displacement field is given by

$$
\mathbf{u}=\sum N_{i} \mathbf{u}_{i}
$$

where $i$ is the index of the grid point, $N_{\mathrm{i}}$ is the shape function with $\Sigma N_{i}=1$.

Because the displacement field jump normal to the fracture, which is a strong discontinuity problem, Eq.(16) could not describe the discontinuous displacement field. Fortunately, the discontinuous displacement field can be approximated by the extended finite element method as

$$
\begin{aligned}
& \mathbf{u}(\mathbf{x})=\sum_{i \in \mathbf{N}} N_{i}(\mathbf{x}) \mathbf{u}_{a i}+\sum_{i \in \mathbb{N}^{\mathrm{errr}}} N_{i}(\mathbf{x})\left(H(\mathbf{x})-H\left(\mathbf{x}_{i}\right)\right) \mathbf{u}_{b i} \\
& +\sum_{i \in \mathrm{N}^{\mathrm{tip}}} N_{i}(\mathbf{x})\left(\psi(\mathbf{x})-\psi\left(\mathbf{x}_{i}\right)\right) \mathbf{u}_{c i}
\end{aligned}
$$

where the nodal set $\mathrm{N}$ is the set of all nodes in the discretized domain, $\mathrm{N}^{\mathrm{enr}}$ is a set of nodes in the elements which are crossed by the fracture, $\mathrm{N}^{\text {tip }}$ is a set of nodes in the crack tip element(as shown in Fig.2), $\mathbf{u}_{a \mathrm{i}}$ are the standard displacement degrees of freedom at node $i, \mathbf{u}_{b \mathrm{i}}$ and $\mathbf{u}_{c \mathrm{i}}$ are the additive degree of freedom for the nodes in the cut elements and tip element, respectively. $H(\mathbf{x})$ is the Heaviside enrichment function as given by Eq.(18), and $H\left(\mathbf{x}_{i}\right)$ is the value of $H(\mathbf{x})$ at the enriched node $\mathrm{i}$

$$
H(\mathbf{x})=\operatorname{sign}(f(\mathbf{x}))=\left\{\begin{array}{cc}
1 & f(\mathbf{x})>0 \\
0 & f(\mathbf{x})=0 \\
-1 & f(\mathbf{x})<0
\end{array}\right.
$$




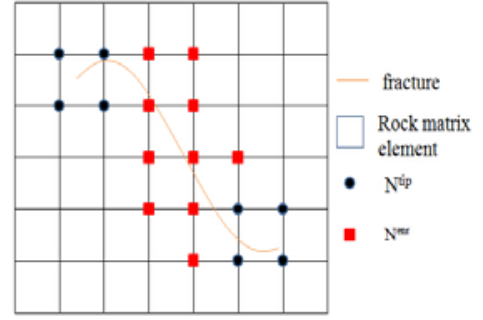

FIGURE II. NODE SELECTION FOR ENRICHMENT FUNCTION

$\psi(\mathbf{x})$ is the near tip asymptotic enrichment function as given by Eq.(19), and $\psi\left(\mathbf{x}_{i}\right)$ is the value of $\psi(\mathbf{x})$ at the enriched node $\mathrm{i}$

$$
\psi(\mathbf{x})=r \sin \frac{\theta}{2}
$$

where $r$ and $\theta$ are the local polar coordinates at the fracture tip.

The fracture width can be derived directly from Eq.(17) by subtracting displacements of the fracture surfaces from each other

$$
w=\left(\mathbf{I}_{\mathrm{f}}^{\mathrm{n}}\right)^{\mathrm{T}} \mathbf{R}\left(2 \sum_{i \in \mathbb{N}^{\text {eir }}} N_{i}(\mathbf{x}) H\left(\mathbf{x}_{i}\right) \mathbf{u}_{b i}+2 \sum_{i \in \mathrm{N}^{\mathrm{tip}}} N_{i}(\mathbf{x}) r \mathbf{u}_{c i}\right)
$$

Symbolically, Eq.(17) can be rewritten as

$$
\mathbf{u}=\mathbf{N}^{\mathrm{u}} \overline{\mathbf{u}}=\mathbf{N}_{a}^{\mathrm{u}} \overline{\mathbf{u}}_{a}+\mathbf{N}_{b}^{\mathrm{u}} \overline{\mathbf{u}}_{b}+\mathbf{N}_{c}^{\mathrm{u}} \overline{\mathbf{u}}_{c}
$$

where $\mathbf{N}_{a}^{\mathrm{u}}$ is the matrix of the standard displacement shape functions, $\mathbf{N}_{b}{ }_{b}$ and $\mathbf{N}_{c}{ }_{c}$ is the matrix of the enriched displacement shape functions for fracture cut elements and tip element, respectively. $\overline{\mathbf{u}}_{a}$ is the vector of the standard displacement degrees of freedom, $\overline{\mathbf{u}}_{b}$ and $\overline{\mathbf{u}}_{c}$ is the vector of the enriched displacement degrees of freedom for fracture cut elements and tip element, respectively.

Accordingly, the strain vector corresponding to the displacement field is given by Eq.(22).

$$
\boldsymbol{\varepsilon}=\mathbf{L} \mathbf{u}=\mathbf{L N}^{\mathrm{u}} \overline{\mathbf{u}}=\mathbf{B}_{a} \overline{\mathbf{u}}_{a}+\mathbf{B}_{b} \overline{\mathbf{u}}_{b}+\mathbf{B}_{c} \overline{\mathbf{u}}_{c}
$$

Similarly, the pressure field is continuous at the fracture surface, while the derivative of pressure (flow rate) is discontinuous, which is a weak discontinuity problem. Therefore, the pressure field can be approximated by the extended finite element method as

$$
\begin{aligned}
& p(\mathbf{x})=\sum_{i \in \mathrm{N}} N_{i}(\mathbf{x}) p_{a i}+\sum_{i \in \mathrm{N}^{\text {elr }}} N_{i}(\mathbf{x})\left(|f(\mathbf{x})|-\left|f\left(\mathbf{x}_{i}\right)\right|\right) p_{b i} \\
& +\sum_{i \in \mathrm{N}^{\text {tip }}} N_{i}(\mathbf{x})\left(\Phi(\mathbf{x})-\Phi\left(\mathbf{x}_{i}\right)\right) p_{c i}
\end{aligned}
$$

where $p_{a \mathrm{i}}$ are the standard pressure degrees of freedom at node $\mathrm{i}, p_{b \mathrm{i}}$ and $p_{c \mathrm{i}}$ are the additive degree of freedom for the nodes in the cut elements and tip element, respectively. $f\left(\mathbf{x}_{i}\right)$ is the value of $f(\mathbf{x})$ at the enriched node i, $\Phi(\mathbf{x})$ is the near tip asymptotic enrichment function as given by Eq.(24), and $\Phi\left(\mathbf{x}_{i}\right)$ is the value of $\Phi(\mathbf{x})$ at the enriched node i.

$$
\Phi(\mathbf{x})=r \cos \frac{\theta}{2}
$$

Symbolically, Eq.(23) can be rewritten as

$$
p=\mathbf{N}^{\mathrm{p}} \overline{\boldsymbol{p}}=\mathbf{N}_{a}^{\mathrm{p}} \overline{\boldsymbol{p}}_{a}+\mathbf{N}_{b}^{\mathrm{p}} \overline{\boldsymbol{p}}_{b}+\mathbf{N}_{c}^{\mathrm{p}} \overline{\boldsymbol{p}}_{c}
$$

where $\mathbf{N}_{a}$ is the matrix of the standard pressure shape functions, $\mathbf{N}_{b}^{\mathrm{u}}{ }_{b}$ and $\mathbf{N}_{c}^{\mathrm{u}}{ }_{c}$ is the matrix of the enriched pressure shape functions for fracture cut elements and tip element, respectively. $\overline{\boldsymbol{p}}_{a}$ is the vector of the standard pressure degrees of freedom, $\overline{\boldsymbol{p}}_{b}$ and $\overline{\boldsymbol{p}}_{c}$ is the vector of the enriched pressure degrees of freedom for fracture cut elements and tip element, respectively.

Substituting Eqs.(2),(21) and (22) into Eq.(12), and according to the irrelevance of the values of $\overline{\mathbf{u}}_{a}, \overline{\mathbf{u}}_{b}$ and $\overline{\mathbf{u}}_{c}$, the following equation is obtained

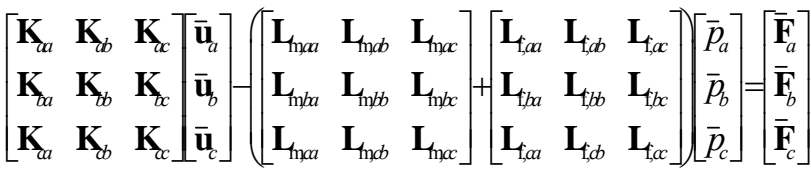

Symbolically, Eq.(26) can be rewritten as

$$
\left[\begin{array}{ll}
\mathbf{K} & -\left(\mathbf{L}_{\mathrm{m}}+\mathbf{L}_{\mathrm{f}}\right)
\end{array}\right]\left[\begin{array}{l}
\overline{\mathbf{u}} \\
\bar{p}
\end{array}\right]+\left[\begin{array}{ll}
0 & 0
\end{array}\right] \frac{\mathrm{d}}{\mathrm{dt}}\left[\begin{array}{c}
\overline{\mathbf{u}} \\
\overline{\mathrm{p}}
\end{array}\right]=\overline{\mathbf{F}}
$$

Substituting Eq.(25) and Eq.(21) into Eq.(15), the XFEM discretization equation of Eq.(15) is gained considering the irrelevance of the values of $\bar{p}_{a}, \bar{p}_{b}$ and $\bar{p}_{c}$

$$
\begin{aligned}
& \left(\left[\begin{array}{lll}
\mathbf{H}_{\mathrm{m}, a a} & \mathbf{H}_{\mathrm{m}, a b} & \mathbf{H}_{\mathrm{m}, a c} \\
\mathbf{H}_{\mathrm{m}, b a} & \mathbf{H}_{\mathrm{m}, b b} & \mathbf{H}_{\mathrm{m}, b c} \\
\mathbf{H}_{\mathrm{m}, c a} & \mathbf{H}_{\mathrm{m}, c b} & \mathbf{H}_{\mathrm{m}, c c}
\end{array}\right]+\left[\begin{array}{lll}
\mathbf{H}_{\mathrm{f}, a a} & \mathbf{H}_{\mathrm{f}, a b} & \mathbf{H}_{\mathrm{f}, a c} \\
\mathbf{H}_{\mathrm{f}, b a} & \mathbf{H}_{\mathrm{f}, b b} & \mathbf{H}_{\mathrm{f}, b c} \\
\mathbf{H}_{\mathrm{f}, c a} & \mathbf{H}_{\mathrm{f}, c b} & \mathbf{H}_{\mathrm{f}, c c}
\end{array}\right]\right)\left[\begin{array}{c}
\bar{p}_{a} \\
\bar{p}_{b} \\
\bar{p}_{c}
\end{array}\right] \\
& +\left(\left[\begin{array}{lll}
\mathbf{C}_{\mathrm{m}, a a} & \mathbf{C}_{\mathrm{m}, a b} & \mathbf{C}_{\mathrm{m}, a c} \\
\mathbf{C}_{\mathrm{m}, b a} & \mathbf{C}_{\mathrm{m}, b b} & \mathbf{C}_{\mathrm{m}, b c} \\
\mathbf{C}_{\mathrm{m}, c a} & \mathbf{C}_{\mathrm{m}, c b} & \mathbf{C}_{\mathrm{m}, c c}
\end{array}\right]+\left[\begin{array}{lll}
\mathbf{C}_{\mathrm{f}, a a} & \mathbf{C}_{\mathrm{f}, a b} & \mathbf{C}_{\mathrm{f}, a c} \\
\mathbf{C}_{\mathrm{f}, b a} & \mathbf{C}_{\mathrm{f}, b b} & \mathbf{C}_{\mathrm{f}, b c} \\
\mathbf{C}_{\mathrm{f}, c a} & \mathbf{C}_{\mathrm{f}, c b} & \mathbf{C}_{\mathrm{f}, c c}
\end{array}\right]\right) \mathrm{d}\left[\begin{array}{c}
\bar{p}_{a} \\
\bar{p}_{b} \\
\bar{p}_{c}
\end{array}\right] \\
& +\left(\left[\begin{array}{lll}
\mathbf{L}_{\mathrm{m}, a a} & \mathbf{L}_{\mathrm{m}, a b} & \mathbf{L}_{\mathrm{m}, a c} \\
\mathbf{L}_{\mathrm{m}, b a} & \mathbf{L}_{\mathrm{m}, b b} & \mathbf{L}_{\mathrm{m}, b c} \\
\mathbf{L}_{\mathrm{m}, c a} & \mathbf{L}_{\mathrm{m}, c b} & \mathbf{L}_{\mathrm{m}, c c}
\end{array}\right]+\left[\begin{array}{lll}
\mathbf{L}_{\mathrm{f}, a a} & \mathbf{L}_{\mathrm{f}, a b} & \mathbf{L}_{\mathrm{f}, a c} \\
\mathbf{L}_{\mathrm{f}, b a} & \mathbf{L}_{\mathrm{f}, b b} & \mathbf{L}_{\mathrm{f}, b c} \\
\mathbf{L}_{\mathrm{f}, c a} & \mathbf{L}_{\mathrm{f}, c b} & \mathbf{L}_{\mathrm{f}, c c}
\end{array}\right]\right) \frac{\mathrm{d}}{\overline{\mathbf{u}}_{a}}\left[\begin{array}{l}
\mathrm{dt} \\
\overline{\mathbf{u}}_{b} \\
\overline{\mathbf{u}}_{c}
\end{array}\right]=\left[\begin{array}{l}
\bar{Q}_{a} \\
\bar{Q}_{b} \\
\bar{Q}_{c}
\end{array}\right]
\end{aligned}
$$

Symbolically, Eq.(28) can be rewritten as 


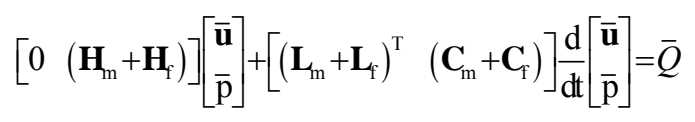

Coupling Eq.(27) and Eq.(29)

$$
\left[\begin{array}{cc}
\mathbf{K} & -\left(\mathbf{L}_{\mathrm{m}}+\mathbf{L}_{\mathrm{r}}\right) \\
0 & \left(\mathbf{H}_{\mathrm{m}}+\mathbf{H}_{\mathrm{f}}\right)
\end{array}\right]\left[\begin{array}{c}
\overline{\mathbf{u}} \\
\overline{\mathrm{p}}
\end{array}\right]+\left[\begin{array}{cc}
0 & 0 \\
\left(\mathbf{L}_{\mathrm{m}}+\mathbf{L}_{\mathrm{f}}\right)^{\mathrm{T}} & \left(\mathbf{C}_{\mathrm{m}}+\mathbf{C}_{\mathrm{f}}\right)
\end{array}\right] \frac{\mathrm{d}}{\overline{\mathrm{dt}}}\left[\begin{array}{c}
\overline{\mathbf{u}} \\
\overline{\mathrm{p}}
\end{array}\right]=\left[\begin{array}{c}
\overline{\mathbf{F}} \\
\bar{Q}
\end{array}\right]
$$

Symbolically, Eq.(30) can be rewritten as

$$
\mathbf{A X}+\mathbf{B} \frac{d \mathbf{X}}{d \mathrm{t}}=\mathbf{C}
$$

In which

$$
\left\{\begin{array}{l}
\mathbf{A}=\left[\begin{array}{cc}
\mathbf{K} & -\left(\mathbf{L}_{\mathrm{m}}+\mathbf{L}_{\mathrm{f}}\right) \\
0 & \left(\mathbf{H}_{\mathrm{m}}+\mathbf{H}_{\mathrm{f}}\right)
\end{array}\right] \\
\mathbf{B}=\left[\begin{array}{cc}
0 & 0 \\
\left(\mathbf{L}_{\mathrm{m}}+\mathbf{L}_{\mathrm{f}}\right)^{\mathrm{T}} & \left(\mathbf{C}_{\mathrm{m}}+\mathbf{C}_{\mathrm{f}}\right)
\end{array}\right] \\
\mathbf{C}=\left[\begin{array}{l}
\overline{\mathbf{F}} \\
\bar{Q}
\end{array}\right] \\
\mathbf{X}=\left[\begin{array}{l}
\overline{\mathbf{u}} \\
\bar{p}
\end{array}\right]
\end{array}\right.
$$

Finally the task is to solve the Eq.(31). In order to keep stability and eliminate the oscillatory effects in the solution, the discretized form of Eq.(31) is as follows:

$$
\left(\mathbf{A}_{t+\Delta t} \Delta t+\mathbf{B}_{t+\Delta t}\right) \mathbf{X}_{t+\Delta t}=\mathbf{B}_{t+\Delta t} \mathbf{X}_{\mathrm{n}}+\mathbf{C}_{t+\Delta t} \Delta t
$$

where $t$ is the time, $\Delta t$ is the time step.

Eq. (33) requires Gauss integration of the parameter over the domain volume, the boundary, and the fracture. In this research, we choose 4-Gauss points in conventional element and 64-Gauss point in cut elements, tip elements, and their neighbours elements[23]. In addition, 6-Gauss points is set along the one-dimensional segments of the fracture delimited with the element edges to integrate the mechanical and mass transfer coupling terms along the fracture.

\section{F. Fracture Propagation and Orientation}

Hydraulic fracture is the mixed mode fracture, the fracture tips will propagate when the equivalent stress intensity factor $K_{\text {e }}$ (expressed as Eq.(34)) is equal to fracture toughness of the rock $K_{\mathrm{IC}}$, and the propagate orientation relies on the maximum circumferential stress criterion so that the expression for fracture growth direction is given by Eq.(35)

$$
K_{\mathrm{e}}=\cos \frac{\theta}{2}\left(K_{\mathrm{I}} \cos ^{2} \frac{\theta}{2}-1.5 K_{\mathrm{II}} \sin \theta\right)
$$

$$
\theta= \begin{cases}0 & \text { if } \quad K_{\mathrm{II}}=0 \\ 2 \arctan \left(\frac{\left.K_{\mathrm{I}} / K_{\mathrm{II}}-\operatorname{sgn}\left(K_{\mathrm{II}}\right) \sqrt{\left(K_{\mathrm{I}} / K_{\mathrm{II}}\right)^{2}+8}\right)}{4}\right) & \text { if } \quad K_{\mathrm{II}} \neq 0\end{cases}
$$

where $K_{\mathrm{I}}$ and $K_{\mathrm{II}}$ are stress intensity factors, that can be obtained by interaction integral.

For general mixed-mode problems, the interaction integral expression and its relationship between the stress intensity factors can be expressed as Eq.(36) and Eq.(37), respectively[24].

$$
\begin{gathered}
I^{(1,2)}=\int_{A}\left[\sigma_{i j}^{(1)} \mathrm{u}_{i, 1}^{(2)}+\sigma_{i j}^{(2)} \mathrm{u}_{i, 1}^{(1)}-W^{(1,2)} \delta_{1 j}\right] q_{, j} d A \\
-\int_{\mathrm{C}^{+} \cup \mathrm{C}^{-}}\left[\sigma_{i 2}^{(1)} \mathrm{u}_{i, 1}^{(2)}+\sigma_{i 2}^{(2)} \mathrm{u}_{i, 1}^{(1)}\right] q m_{2} d \Gamma \\
I^{(1,2)}=\frac{2}{E^{*}}\left(K_{\mathrm{I}}^{(1)} K_{\mathrm{I}}^{(2)}+K_{\mathrm{II}}^{(1)} K_{\mathrm{II}}^{(2)}\right)
\end{gathered}
$$

where superscript 1 and 2 represents two states, state 1 corresponds to the present state and state 2 is an auxiliary state which will be chosen as the asymptotic fields for Mode I or Mode II[24], $q_{, j}$ is the derivative of the weight function $q$ with respect to local $x_{j}$-axis, the region $A$ and contour $\mathrm{C}^{+} \cup \mathrm{C}^{-}$as shown in Fig. 3, $W^{(1,2)}$ is the interaction energy expressed as Eq.(38), $m_{2}$ is the 2 nd component of the outward normal vector to the closed contour $\mathrm{C}^{+} \cup \mathrm{C}^{-} \cup \mathrm{C}^{\mathrm{o}}, E^{*}=\mathrm{E}$ for plane stress and $E^{*}=\mathrm{E} /\left(1-v^{2}\right)$ for plane strain ( $E$ is Young's modulus, $v$ is the Poisson's ratio).

$$
W^{(1,2)}=\sigma_{i j}^{(1)} \varepsilon_{i j}^{(2)}=\sigma_{i j}^{(2)} \varepsilon_{i j}^{(1)}
$$

Choosing the auxiliary state as the pure mode I asymptotic field (i.e., $K_{\mathrm{I}}^{(2)}=1, K_{\mathrm{II}}^{(2)}=0$ ) and pure mode II asymptotic field (i.e., $K_{\mathrm{I}}^{(2)}=1, K_{\mathrm{II}}^{(2)}=0$ ), respectively, $K_{\mathrm{I}}$ and $K_{\text {II }}$ can be expressed as follows

$$
\begin{gathered}
K_{\mathrm{I}}=\frac{E^{*}}{2} I^{(1, \text { Mode I })} \\
K_{\text {II }}=\frac{E^{*}}{2} I^{(1, \text { Mode II })}
\end{gathered}
$$

The details for derivation can be found in Dolbow et al.[24].

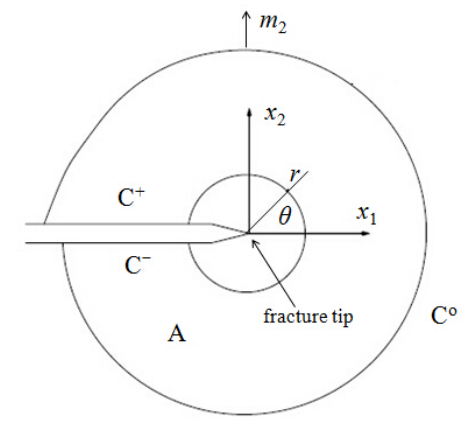

FIGURE III. THE CONTOUR AND DOMAIN TO COMPUTE THE INTERACTION INTEGRAL AND LOCAL COORDINATE SYSTEM AT THE FRACTURE TIP 


\section{MODEL VALIDATION}

Because the hydraulic fracturing is a complicated problem, no exactly solution could be found to verify our numerical model except for the simplest examples. Therefore the results obtained from the numerical simulation and simplified semianalytical(KGD) solution are presented in this part. This can be considered as verification of our model. The analytical expression for fracture length and fracture opening at the wellbore given as function of time $t$ as [16]

$$
\begin{gathered}
L_{\mathrm{f}}(t)=0.68\left[\frac{E q^{3}}{2\left(1-v^{2}\right) \mu}\right]^{1 / 6} t^{2 / 3} \\
w(0, t)=1.87\left[\frac{2\left(1-v^{2}\right) \mu q^{3}}{E}\right]^{1 / 6} t^{1 / 3}
\end{gathered}
$$

where $q$ is the injection rate per unit height of the fracture $\left(\mathrm{m}^{2} / \mathrm{s}\right)$.

Consider a 40 meters long and 50 meters wide computational domain meshed by the $200 \times 250$ structured grid, the geometry and boundary condition of the example are depicted in Fig.4, in this example a fracture with an initial length of 0.5 meters is driven by the injection of a Newtonian fluid at the constant flow rate of $0.001 \mathrm{~m}^{2} / \mathrm{s}$, young's modulus is $20 \mathrm{GPa}$, Poisson's ratio is 0.19 , fracture toughness of the rock is $1.5 \mathrm{MPa} \cdot \mathrm{m}^{1 / 2}$.

Fig. 5 shows a comparison between the fracture length obtained from the numerical solutions and that obtained from the semi-analytical solutions. Good agreement between numerical predictions and semi-analytical solutions, and the maximal relative error is $4.1 \%$. The XFEM model predicts a slight smaller fracture length than KGD model at the same injection time, the reason for this is that KGD neglecting leakoff effect. Fig. 6 makes a comparison of the fracture width at the wellbore between the numerical and the semi-analytical solutions. Both computational results fit well with each other, the maximal relative error is $10 \%$ at the beginning of the injection, but the relative error is under $5 \%$ as the injection time increase. In other words, Fig.5 and 6 proved that the XFEM model has ability to model fracture propagation through fluid injection.

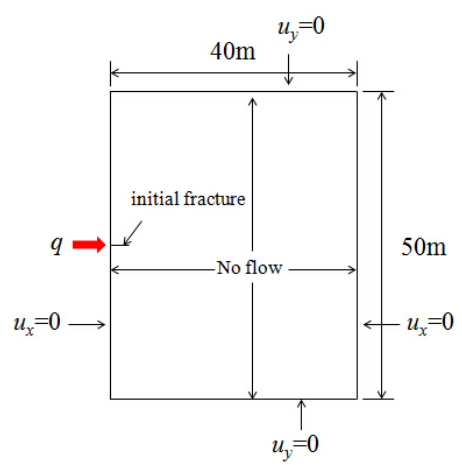

FIGURE IV. THE GEOMETRY AND BOUNDARY CONDITION OF THE VERIFICATION EXAMPLE

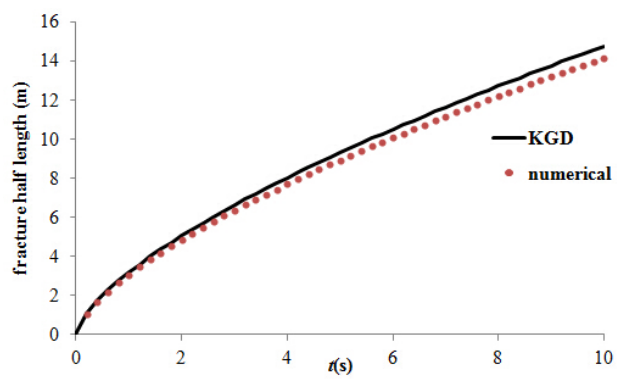

FIGURE V. FRACTURE LENGTH VERSUS INJECTION TIME, COMPUTED WITH NUMERICAL AND SEMI-ANALYTICAL (KGD) MODELS

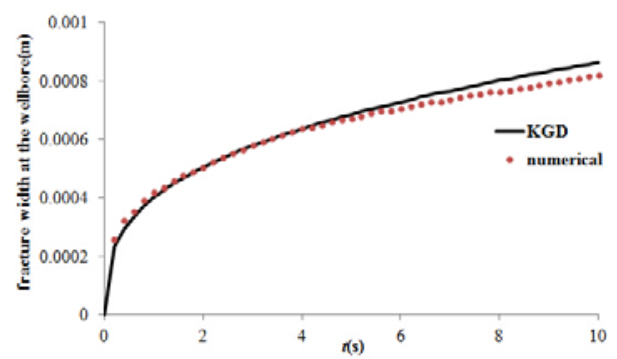

FIGURE VI. FRACTURE WIDTH AT THE WELLBORE VERSUS INJECTION TIME, COMPUTED WITH NUMERICAL AND SEMIANALYTICAL (KGD) MODELS

\section{NUMERICAL EXAMPLE}

In this section, we simulate fractures propagate simultaneously from three perforating clusters in the horizontal well. In the process of multi cluster fracturing, the flow distribution in the well is a dynamic process. When ignoring wellbore storage effects, the total injection rate is equal to the sum of the flow rate in all cluster

$$
Q(\mathrm{t})=\sum_{i=1}^{N} q_{i}(\mathrm{t})
$$

where $q_{i}(\mathrm{t})$ is the injection rate in No. i fracture $\left(\mathrm{m}^{3} / \mathrm{s}\right), N$ is the fracture number, $Q(\mathrm{t})$ is the total injection $\operatorname{rate}\left(\mathrm{m}^{3} / \mathrm{s}\right)$.

By ignoring the wellbore frictional pressure drop, and according to the Kirchhoff's second law, the fluid pressure in the wellbore heel $\left(p_{0}\right)$ is equal to the sum of perforation frictional pressure $\operatorname{drop}\left(p_{p f, i}\right)$ and pressure at the fracture $\operatorname{mouth}\left(p_{w, i}\right)$

$$
p_{0}=p_{w, i}+p_{p f, i}
$$

and $[25]$

$$
p_{p f, i}=\frac{2.2326 \times 10^{-4} q_{\mathrm{i}}^{2} \rho}{n^{2} d^{4} \mathrm{C}^{2}}
$$

where $\rho$ is the density of fracturing fluid $\left(\mathrm{kg} / \mathrm{m}^{3}\right), n$ is the number of perforation, $d$ is the perforation diameter(mm), C is the flow coefficient. 
This XFEM model has no limitations to calculate a large scale problem, however, in order to reduce the computational time, a small scale problem are considered. The fractures are assumed to be symmetrical about the wellbore, so only half of the fractures are modeled. Fig.7 shows the geometric model with a dimension of $25 \mathrm{~m} \times 120 \mathrm{~m}$, three hydraulic fractures with initial lengths of $1 \mathrm{~m}$ at $20 \mathrm{~m}$ cluster space are considered. The fracture height is $50 \mathrm{~m}$. A far-field stress of $-100 \mathrm{MPa}$ is applied on the right boundary, the displacement in $\mathrm{X}$ direction is 0 on the left boundary, and $-70 \mathrm{MPa}$ is applied on the top and bottom boundaries, negative value of stress indicates compression. Besides the pore pressure at boundary is set to be constant with the value of the initial pore pressure $50 \mathrm{MPa}$. In the following discussion, fracture 1,2 and 3 represent the fractures which propagate from the bottom, middle and top cluster respectively as shown in Fig.7.

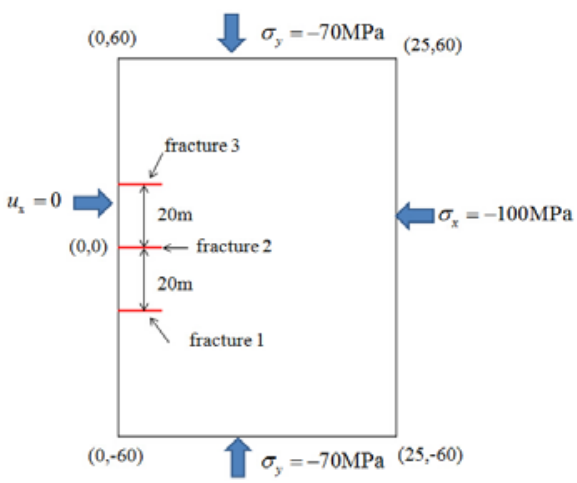

FIGURE VII. THE GEOMETRY AND BOUNDARY CONDITION OF THE MULTI-CLUSTER FRACTURING EXAMPLE

The displacement in the y-direction by the end of 10 seconds' injection is shown in Fig.8. It is obvious that the displacement in the y-direction is discontinuous in the fracture. On the upper fracture surface, it is positive and on the lower face negative, it means the fracture is open.

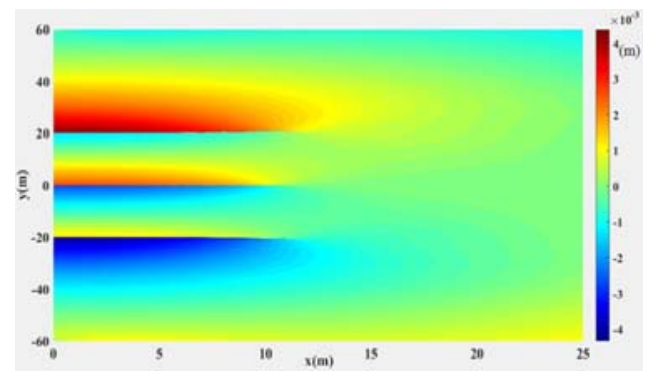

FIGURE VIII. DISPLACEMENT IN Y-DIRECTION OF 20 M CLUSTER SPACE CASE

The contours of rock stress in y-direction by the end of injection is shown in Fig.9. From Fig.9 we can infer that a high tensile stress area existing in front of the fracture tips dues to the tip singularity. And the rock among the fractures is compressed because the fluid pressure within the fracture directly squeeze the rock in y-direction

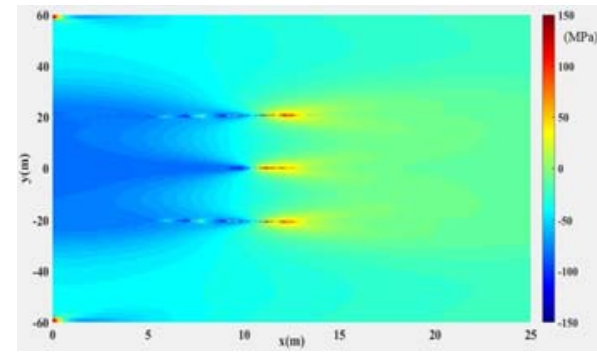

FIGURE IX. ROCK STRESS IN Y-DIRECTION OF 20 M CLUSTER SPACE CASE

Fracture width variation curves along fracture length by the end of injection is shown in Fig.10. As it can be seen, the width of fracture 1 and 3 is almost the same because of symmetrical characteristic of this model. The width curve of fracture 2 has a very good streamline property, whereas the width curves of fracture 1 and fracture 3 are shaped like the sawtooth. This is because fracture 2 approximately extend flatly, whlie fracture 1 and fracture 3 bending propagate. The fracture width may have a local minima value at the fracture inflection point, which is detrimental to proppant transportation in fracture, and can lead to proppant screenout.

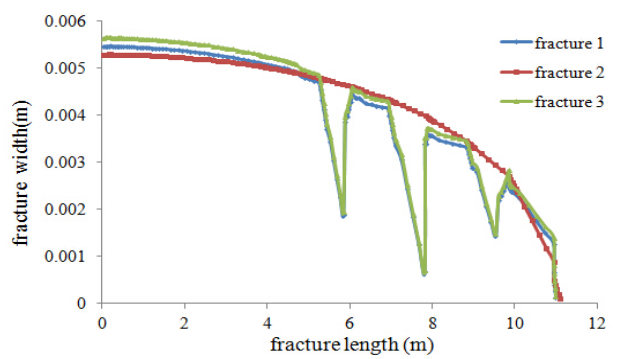

FIGURE X. WIDTH OF FRACTURE 1, 2 AND 3 OF 20 M CLUSTER SPACE CASE

Fig. 11 show the pressure contours of the formation by the end of injection. As it can be seen, the fluid pressure in the fracture is higher than that in the matrix. Because the matrix permeability is very low, the pore fluid pressure at most areas of the formation remains $50 \mathrm{MPa}$ (the initial pore pressure), and only in a very small area around the fracture the fluid pressure increase because of the filtration of fracturing fluid.

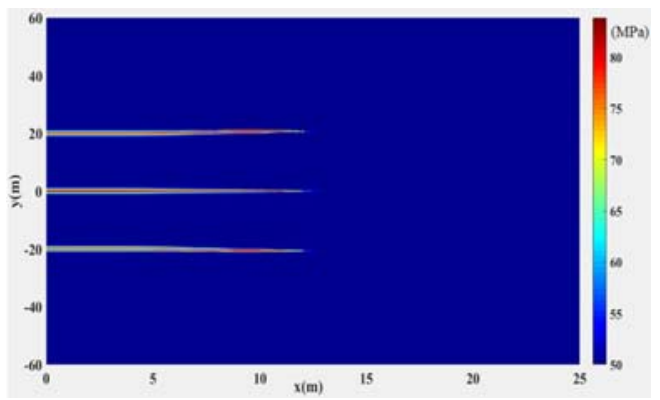

FIGURE XI. FORMATION FLUID PRESSURE OF 20 M CLUSTER SPACE CASE

\section{CONCLUSIONS}

In the present paper, an improved approach to simulate hydraulic fracturing has been presented. The fully coupled 
formulation was established based upon the flow continuity equation and the force equilibrium equation, which described the fluid flow along the fracture, flow through the fracture sides toward the surrounding porous medium and flow in the porous medium as well as the solid skeleton deformation. The fully coupled formulation was solved by the extended finite element method(XFEM). In comparison with the existing models for hydraulic fracturing, this approach has the following advantages: (1)No need to adopt leak-off coefficient to describe the fluid leak-off phenomenon;(2)No need to assume fracture propagation orientation;(3)No need to conform fracture sides with the element boundary and use special element near the crack tips; (4)After fracture propagation, remeshing is not a necessity. A comparison of the model numerical solutions with the semi-analytical solution indicates that the model is valid. Fractures simultaneously propagate from three perforating clusters in the horizontal well is simulated at the end of this paper.

\section{ACKNOWLEDGMENT}

The research is supported by the National Science and Technology Major Project of China (2016ZX05002-005-0011), Open funds of State Key Laboratory of shale oil gas accumulation mechanism and effective development (G580015-ZS-WX042)

\section{REFERENCES}

[1] Sasaki, S. (1998) Characteristics of microseismic events induced during hydraulic fracturing experiments at the Hijiori hot dry rock geothermal energy site, Yamagata,Japan. Tectonophysics 289: 171-188.

[2] Berumen, S., Tiab, D. and F. Rodriguez (2000) Constant rate solutions for a fractured well with an asymmetric fracture. Journal of Petroleum Science and Engineering 25: 49-58

[3] Hayashi, K., A. Sato, and T. Ito (1997) In situ stress measurements by hydraulic fracturing for a rock mass with many planes of weakness. International Journal of Rock Mechanics \& Mining Sciences 34: 45-58.

[4] Raaen, A.M., Skomedal, E., Kjorholt, H., Markestad, P. and Okland, D. (2001) Stress determination from hydraulic fracturing tests: The system stiffness approach. International Journal of Rock Mechanics and Mining Sciences 38: 529-541.

[5] Belytschko T, Black T (1999) Elastic crack growth in finite elements with minimal remeshing. Int J Numer Meth Eng 45:601-620.

[6] Li YM, Jiang YS, Zhao JZ, Liu CY, and Zhang LH (2015) Extended finite element method for analysis of multi-scale flow in fractured shale gas reservoirs. Environ Earth Sci 73: 6035-6045.

[7] Brice Lecampion(2009) An extended finite element method for hydraulic fracture problems. Commun. Numer. Meth. Engng 25:121133

[8] Lamb A.R., Gerard J. Gorman, Derek Elsworth(2013) A fracture mapping and extended finite element scheme for coupled deformation and fluid flow in fractured porous media. Int. J. Numer. Anal. Meth. Geomech 37:2916-2936.

[9] REN QW, DONG YW,YU TT (2009) Numerical modeling of concrete hydraulic fracturing with extended finite element method. Science in China. Series E: Technological Sciences 52: 559-565.

[10] Gordeliy E, Peirce A (2013) Coupling schemes for modeling hydraulic fracture propagation using the XFEM. Comput. Methods Appl. Mech. Engrg 253:305-322.

[11] Dahi-Taleghani, A (2009) Analysis of hydraulic fracture propagation in fractured reservoirs: an improved model for the interaction between induced and natural fractures. Ph. D Thesis, The University of Texas at Austin.

[12] Zhou L, Gou Y, Hou ZM, Were P (2015) Numerical modeling and investigation of fracture propagation with arbitrary orientation through fluid injection in tight gas reservoirs with combined XFEM and FVM. Environ Earth Sci 73: 5801-5813.

[13] Wang XL, Liu C, Wang H, Liu H, Wu HA (2016) Comparison of consecutive and alternate hydraulic fracturing in horizontal wells using XFEM-based cohesive zone method. Journal of Petroleum Science and Engineering 143:14-25.

[14] Carrier B, Granet S (2012) Numerical modeling of hydraulic fracture problem in permeable medium using cohesive zone model. Engineering Fracture Mechanics 79: 312-328.

[15] Mohammadnejad.T , Khoei A. R. (2013a) Hydro-mechanical modeling of cohesive crack propagation in multiphase porous media using the extended finite element method. Int. J. Numer. Anal. Meth. Geomech $37: 1247-1279$.

[16] Mohammadnejad.T, Khoei A. R. (2013b)An extended finite element method for hydraulic fracture propagation in deformable porous media with the cohesive crack model. Finite Elements in Analysis and Design73:77-95.

[17] Meschke. G, Leonhart. D (2015) A Generalized Finite Element Method for hydro-mechanically coupled analysis of hydraulic fracturing problems using space-time variant enrichment functions. Comput. Methods Appl. Mech. Engrg. 290 (2015) 438-465.

[18] Dahi-Taleghani, A and Olson, J.E (2011) Numerical Modeling of Multistranded-Hydraulic-Fracture Propagation: Accounting for the Interaction Between Induced and Natural Fractures. SPE Journal 16: 575-581.

[19] Dahi-Taleghani, A and Olson, J.E (2014) How Natural Fractures Could Affect Hydraulic-Fracture Geometry. SPE Journal 19:161-171.

[20] Khoei A. R., M. Hirmand, M. Vahab and M. Bazargan(2015) An enriched FEM technique for modeling hydraulically driven cohesive fracture propagation in impermeable media with frictional natural faults: Numerical and experimental investigations. Int. J. Numer. Meth. Engng 104:439-468.

[21] Jiang YS, Zhao JZ, Li YM, Jia H, and Zhang LH (2014) Extended Finite Element Method for Predicting Productivity of Multifractured Horizontal Wells. Mathematical Problems in Engineering 2014:1-9.

[22] Fei Liu, Li-qiang Zhao, Ping-li Liu, Zhi-feng Luo, Nian-yin Li, and Peishan Wang(2015) An Extended Finite Element Model for Fluid Flow in Fractured Porous Media. Mathematical Problems in Engineering 2015:1-10.

[23] B. Prabel, A. Combescure, A. Gravouil and S. Marie(2007) Level set XFEM non-matching meshes: Application to dynamic crack propagation in elastic-plastic media. Int. J. Numer. Meth. Engng 69:1553-1569.

[24] Dolbow J,Nicolas Moësb,Ted Belytschkoc(2001) An extended finite element method for modeling crack growth with frictional contact. Comput. Methods Appl. Mech. Engrg 190:6825-6846.

[25] LU Yanjun, YANG Zhaozhong, LI Xiaogang, HUANG Junping, ZHANG Shide(2014) Phased Fracturing Technology and Field Applications for Open Hole Horizontal Wells. XINJIANG PETROLEUM GEOLOGY 35:230-234. 